\title{
Pythagorean Triangle and Special Pyramidal Numbers
}

\author{
M. A. Gopalan ${ }^{1}$, V. Sangeetha ${ }^{2}$, Manju Somanath ${ }^{3}$ \\ ${ }^{1}$ Department of Mathematics, Srimathi Indira Gandhi College,Trichy-2,India \\ ${ }^{2,3}$ Department of Mathematics, National College, Trichy-1,India
}

Abstract: Patterns of Pythagorean triangle, where, in each of which either a leg or the hypotenuse is a pentagonal pyramidal number and Centered hexagonal pyramidal number, in turn are presented.

Keywords: Pythagorean triangles, pentagonal pyramidal,centered hexagonal pyramidal.

\section{Introduction}

The method of obtaining three non-zero integers $\alpha, \beta$ and $\gamma$ under certain relations satisfying the equation $\alpha^{2}+\beta^{2}=\gamma^{2}$ has been a matter of interest to various mathematicians [1,2,3].In [4-12], special Pythagorean problems are studied.In this communication, we present yet another interesting Pythagorean problem. That is, we search for patterns of Pythagorean triangles where in each of which, either a leg or the hypotenuse is represented by a pentagonal pyramidal number and centered hexagonal pyramidal number, in turn.

$P_{n}^{m}$ - m-gonal pyramidal number of rank $\mathrm{n}$

\section{Notation}

$C P_{n}^{m}$ - centered m-gonal pyramidal number of rank $\mathrm{n}$

$t_{m, n}$ - polygonal number of rank $\mathrm{n}$.

\section{Method of Analysis}

Let $(m, n, k)$ represent a triple of non-zero distinct positive integers such that

$$
m=(k+1) n
$$

Let $P(\alpha, \beta, \gamma)$ be the Pythagorean triangle whose generators are $m, n$. Consider

$$
\alpha=2 m n ; \beta=m^{2}-n^{2} ; \gamma=m^{2}+n^{2} \text {. }
$$

It is observed that, for suitable choices of $\mathrm{n}$, either a leg or hypotenuse of the Pythagorean triangle $\mathrm{P}$ is represented by a pentagonal pyramidal number and centered hexagonal pyramidal number , in turn.Different choices of $\mathrm{n}$ along with the corresponding sides of the Pythagorean triangle are illustrated below

Choice 3.1

$$
\text { Let } n=4 k+3 \text {. }
$$

The corresponding sides of the Pythagorean triangle are

Note that $\alpha=P_{n}^{5}$

$$
\begin{aligned}
& \alpha=32 k^{3}+80 k^{2}+66 k+18 \\
& \beta=16 k^{4}+56 k^{3}+57 k^{2}+18 k \\
& \gamma=16 k^{4}+56 k^{3}+89 k^{2}+66 k+18
\end{aligned}
$$

Choice 3.2

$$
\text { Let } n=2 k^{2}+4 k+3
$$

The corresponding sides of the Pythagorean triangle are

Note that $\gamma=P_{n}^{5}$

$$
\begin{aligned}
& \alpha=8 k^{5}+40 k^{4}+88 k^{3}+104 k^{2}+66 k+18 \\
& \beta=4 k^{6}+24 k^{5}+60 k^{4}+80 k^{3}+57 k^{2}+18 k \\
& \gamma=4 k^{6}+24 k^{5}+68 k^{4}+112 k^{3}+113 k^{2}+66 k+18
\end{aligned}
$$

Note

It is worth mentioning here that, for the following two choices of $\mathrm{m}, \mathrm{n}$ given by (i) $n=4 k, m=k(n+1)$ and (ii) $n=2 k^{3}-3, m=k n$ the sides $\alpha$ and $\beta$ represent $P_{n}^{5}$ respectively.

Choice 3.3

$$
\text { Let } n=2(k+1)
$$

The corresponding sides of the Pythagorean triangle are

$$
\begin{aligned}
\alpha & =8 k^{3}+24 k^{2}+24 k+8 \\
\beta & =4 k^{4}+16 k^{3}+20 k^{2}+8 k \\
\gamma & =4 k^{4}+16 k^{3}+28 k^{2}+24 k+8 \\
\text { Note that } \alpha=C P_{n}^{6} &
\end{aligned}
$$


Choice 3.4

$$
\text { Let } n=k(k+2)
$$

The corresponding sides of the Pythagorean triangle are

Note that $\beta=C P_{n}^{6}$

$$
\begin{aligned}
& \alpha=2 k^{5}+10 k^{4}+16 k^{3}+8 k^{2} \\
& \beta=k^{6}+6 k^{5}+12 k^{4}+8 k^{3} \\
& \gamma=k^{6}+6 k^{5}+14 k^{4}+16 k^{3}+8 k^{2}
\end{aligned}
$$

Choice 3.5

$$
\text { Let } n=k^{2}+2 k+2
$$

The corresponding sides of the Pythagorean triangle are

Note that $\gamma=C P_{n}^{6}$

$$
\begin{aligned}
& \alpha=2 k^{5}+10 k^{4}+24 k^{3}+32 k^{2}+24 k+8 \\
& \beta=k^{6}+6 k^{5}+16 k^{4}+24 k^{3}+20 k^{2}+8 k \\
& \gamma=k^{6}+6 k^{5}+18 k^{4}+32 k^{3}+36 k^{2}+24 k+8
\end{aligned}
$$

Properties

(1) $3(\gamma-\beta)$ is a Nasty Number.

(2) $\frac{\alpha \beta}{12 p_{k}^{3}}$ is a biquadratic integer.

(3) $\frac{\alpha \beta}{P_{k}^{5}+t_{3, k}}$ is a perfect square.

(4) $\frac{\gamma}{\beta}=\frac{C P_{k+1}^{3}}{P_{k}^{5}+2 t_{3, k}}$

(5) $\quad \alpha$ is a perfect square when $k=2 p^{2}-1$

(6) $6(\gamma-\alpha)$ is a Nasty number.

(7) $\frac{\gamma \alpha}{C P_{k+1}^{3}}$ is a biquadratic integer.

(8) $\quad \frac{3 \gamma}{\beta}=\frac{C P_{k+1}^{3}}{P_{k}^{3}}$

\section{Conclusion}

One may search for other patterns of Pythagorean triangles, where, in each of which either a leg or the hypotenuse is represented by other polygonal and pyramidal numbers.

\section{References}

[1]. L.E.Dickson, History of Theory of Numbers, Vol.2, Chelsea Publishing Company,New York, 1971.

[2]. L.J.Mordell, Diophantine Equations, Academic Press, New York, 1969.

[3]. S.B.Malik, "Basic Number Theory", Vikas Publishing House Pvt. Limited,New Delhi,1998.

[4]. B.L.Bhatia and Suriya Mohanty,Nasty Numbers and their Characterization, Mathematical Education,1985 pp.34-37.

[5]. M.A.Gopalan and S.Devibala, Pythagorean Triangle: A Treasure House, Proceeding of the KMA National Seminar on Algebra,Number Theory and Applications to Coding and Cryptanalysis, Little Flower College, Guruvayur.Sep.(2004) 16-18.

[6]. M.A.Gopalan and R.Anbuselvi,A Special Pythagorean Triangle, Acta Cienia Indica,XXXI M (1) (2005) pp.53.

[7]. M.A.Gopalan and S.Devibala, On a Pythagorean Problem,Acta Cienia Indica,XXXIM(4) (2006) pp.1451.

[8]. M.A.Gopalan and S.Leelavathi,Pythagorean Triangle with Area/Perimeter as a Square Integer, International Journal of Mathematics, Computer Science and Information Technology 1(2) (2008) 199-204.

[9]. M.A.Gopalan and S.Leelavathi,Pythagorean Triangle with 2Area/Perimeter as a Cubic Integer,Bulletin of Pure and Applied Sciences 26 E(2) (2007) 197-200.

[10]. M.A.Gopalan and A.Gnanam,Pairs of Pythagorean Triangles with Equal Perimeters,Impact J.Sci.Tech 1(2) (2007) 67-70.

[11]. M.A.Gopalan and S.Devibala, Pythagorean Triangle with Triangular Number as a Leg, Impact J.Sci.Tech 2(3) (2008) 135-138

[12]. M.A.Gopalan and G.Janaki,Pythagorean Triangle with Nasty Number as a Leg,Journal of Applied Mathematical Analysis and Applications 4 (1-2) (2008) 13-17.

[13]. M.A.Gopalan, V.Sangeetha and Manju Somanath, Pythagorean Triangle and Pentagonal Number ,Accepted for publication in Cayley Journal of Mahematics. 